
ecological oscillators 



\title{
Dispersal and noise: Various modes of synchrony in ecological oscillators
}

\author{
Paul C. Bressloff and Yi Ming Lai
}

the date of receipt and acceptance should be inserted later

\begin{abstract}
We use the theory of noise-induced phase synchronization to analyze the effects of dispersal on the synchronization of a pair of predator-prey systems within a fluctuating environment (Moran effect). Assuming that each isolated local population acts as a limit cycle oscillator in the deterministic limit, we use phase reduction and averaging methods to derive a Fokker-Planck equation describing the evolution of the probability density for pairwise phase differences between the oscillators. In the case of common environmental noise, the oscillators ultimately synchronize. However the approach to synchrony depends on whether or not dispersal in the absence of noise supports any stable asynchronous states. We also show how the combination of correlated (shared) and uncorrelated (unshared) noise with dispersal can lead to a multistable steady-state probability density.
\end{abstract}

\section{Introduction}

An important problem in ecology is understanding the mechanisms for synchronizing spatially separated populations or patches that constitute a metapopulation. It is a crucial factor in conservation because synchrony is strongly correlated with the chances of global extinction [14]. Conversely, synchrony can help to eliminate an outbreak in pest or pathogen control. For example, work on measles has shown that although vaccination reduces the size of an epidemic, it can also desynchronize populations thus promoting persistence $[3,33]$. There are two basic mechanisms for synchronizing patches within a metapopulation [21]: isolated patches can be driven by the same environmental fluctuations (the so-called Moran effect $[28,15]$ ) or patches can interact

Paul C. Bressloff

Department of Mathematics, University of Utah, Salt Lake City, UT 84112 USA E-mail: bressloff@math.utah.edu

Yi Ming Lai

Mathematical Institute, University of Oxford, Oxford, UK E-mail: lai@maths.ox.ac.uk 
with each other through dispersal in a constant environment $[30,16,2,40]$. The dominant mechanism will depend on the spatial scale of the metapopulation and the nature of the local patch dynamics. An oscillating patch is often modeled as a limit cycle solution of a system of ordinary differential equations, where the timing along each limit cycle is specified in terms of a single phase variable. The phase-reduction method can then be used to analyze synchronization of an ensemble of oscillators by approximating the high-dimensional limit cycle dynamics as a closed system of equations for the corresponding phase variables [19,4]. Within the context of ecology, Goldwyn and Hastings have used the theory of weakly coupled phase oscillators to investigate various modes of synchronous and asynchronous phase-locking in predator-prey systems weakly coupled by dispersal $[11,12]$. They have also examined the joint effects of dispersal and environmental fluctuations, by simulating ensembles of predator-prey oscillators with diffusive or global coupling and spatially correlated Poisson inputs [13]; each predator-prey patch is described by the Rosenzweig and MacArthur (RM) model $[35,17]$

Recently, a complementary approach to analyzing the effects of external fluctuations on the synchronization of predator-prey populations has been developed by Lai et. al. [20], based on the theory of noise-induced phase synchronization $[31,37,10,29,26,38,22]$. The latter is an extension of phase-reduction methods to stochastic limit cycle oscillators that provides an analytical framework for studying the synchronisation of an ensemble of oscillators driven by a common randomly fluctuating input; in the case of the Moran effect such an input would be due to environmental fluctuations. Interestingly, noise-induced phase synchronization appears to occur in other areas of biology. For example, evidence for such a mechanism has been found in experimental studies of the olfactory bulb [5]. It is also suggested by the related phenomenon of spike-time reliability, in which the reproducibility of a single neuron's output spike train across trials is greatly enhanced by a fluctuating input when compared to a constant input $[25,6]$. In our previous work [20], we assumed that the predator-prey oscillators were uncoupled (no dispersal). However, we took into account the effects of both correlated and uncorrelated noise sources: environmental fluctuations treated as a common extrinsic noise source, and uncorrelated demographic noise arising from finite size effects. (See [1] for a related study of noise-induced phase synchronisation in an ensemble of neural population oscillators). We incorporated demographic noise using a stochastic urn model [23]. Approximating the associated master equation using a Kramers-Moyal expansion [9], we then derived a Langevin equation for an ensemble of predator-prey systems. We showed that the multiplicative Gaussian noise terms could be decomposed into a set of independent white noise processes that were uncorrelated across populations (demographic noise), and an additional white noise term that was common to all populations (environmental noise). Assuming that each predator-prey system acted as a limit cycle oscillator in the deterministic limit, we used phase reduction and averaging methods to derive the steady state probability density for pairwise phase dif- 
ferences between oscillators, which was then used to determine the degree of synchronization of the metapopulation.

In this paper, we extend our previous work to the coupled RM model of Goldwyn and Hastings [13]. We show how the theory of stochastic differential equations and noise-induced phase synchronisation can be used to develop an analytical framework for understanding the combined effects of dispersal and noise, at least in the case of two patches. We begin by neglecting the effects of demographic noise (section 2). As previously shown by Goldwyn and Hastings [11,13], there exist parameter regimes where dispersal alone supports both synchronous and asynchronous phase-locked states. Here we show how the existence of an asynchronous state effects the approach to synchrony when common external fluctuations are included. That is, when dispersal supports only synchronous phase-locking, the time-dependent probability density function (PDF) is Gaussian-like with a width that first increases and then decreases as it moves towards the synchronous state. Moreover, the rate of stochastic synchronization varies approximately linearly with the initial phase difference between the two oscillators. On the other hand, if dispersal also supports asynchronous phase-locking, then for a range of initial conditions the deterministic system converges to the asynchronous state; environmental noise is then required to shift the system out of the basin of attraction of the asynchronous state. This results in a much broader PDF at intermediate times, which exhibits a bimodal structure before ultimately converging to the synchronous state. Consequently, there is a sharp decrease in the rate of stochastic synchronisation as the initial phase difference increases from zero. We interpret such behaviour in terms of analytical solutions to a Fokker-Planck equation that determines the PDF of the pair-wise phase difference of the oscillators. We then investigate the effects of uncorrelated demographic noise on the steadystate PDF, which in the absence of uncorrelated noise reduces to a Dirac delta function at zero phase difference (section 3). In the case where dispersal and correlated environmental noise both cause synchronous phase-locking, uncorrelated noise leads to a unimodal steady-state PDF centered about the synchronous state, which implies that there is a reduction in the degree of synchrony. On the other hand, when dispersal and correlated noise generate transient peaks at asynchronous states, before eventually converging to the correlated noise-induced synchronous state, the uncorrelated noise can cause these transient peaks to become persistent so that the steady-state PDF exhibits multistability.

\section{Dispersal and the Moran effect}

\subsection{The Rosenzweig-Macarthur Model}

We consider a predator-prey system consisting of $N$ identical patches labeled $i=1, \ldots, N$. In the absence of dispersal or external perturbations, the intrinsic deterministic dynamics of each patch is described by a canonical ecologi- 

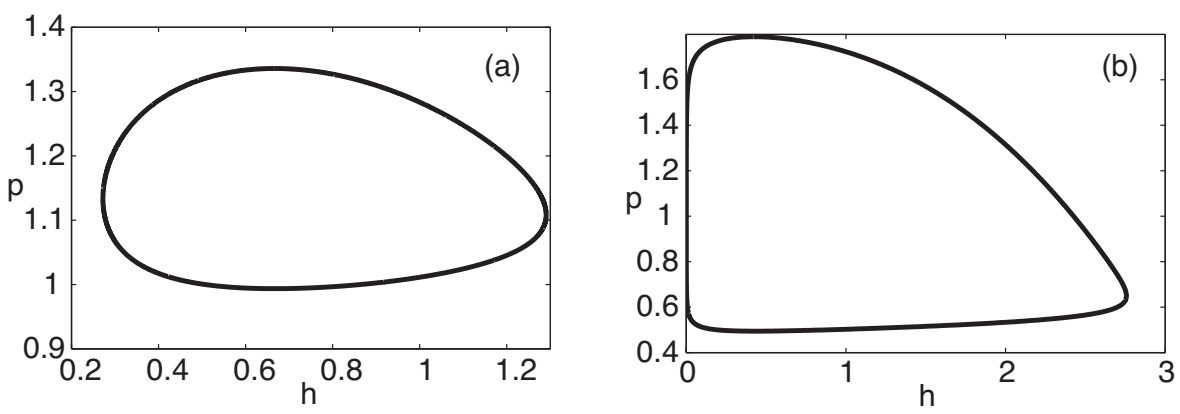

Fig. 1 Limit cycles in the h-p plane for an isolated RM patch. (a) $\epsilon=0.1, \alpha=0.4, \eta=0.4$. (b) $\epsilon=0.1, \alpha=0.3, \eta=0.3$.

cal model known to exhibit limit cycle oscillations, namely, the RosenzweigMacarthur (RM) model [35,17]. For a single isolated patch, the dynamics is given by the pair of equations

$$
\begin{aligned}
\frac{d h_{i}}{d t} & =r h_{i}\left(1-\frac{h_{i}}{K}\right)-\frac{c a p_{i} h_{i}}{b+h_{i}} \\
\frac{d p_{i}}{d t} & =\frac{a p_{i} h_{i}}{b+h_{i}}-m p_{i}
\end{aligned}
$$

where $h_{i}$ represents the population of prey (or herbivores) and $p_{i}$ the population of predators. The RM model assumes logistic growth of prey with rate $r$ up to a carrying capacity $K$, linear predator mortality of rate $m$, and a Holling type-II (or Michaelis-Menten) functional response with parameters $a, b$. The loss of prey due to predation also depends linearly on $c$ with $c>1$, which implies that the loss in prey population due to predation is faster than the gain in predators. Equations (1) can be nondimensionalised as in [11] to obtain, for the $i$-th population:

$$
\begin{aligned}
\frac{d h_{i}}{d \tau} & =\frac{1}{\epsilon}\left(h_{i}\left(1-\alpha h_{i}\right)-\frac{p_{i} h_{i}}{1+h_{i}}\right) \equiv F_{h}\left(h_{i}, p_{i}\right) \\
\frac{d p_{i}}{d \tau} & =\frac{p_{i} h_{i}}{1+h_{i}}-\eta p_{i} \equiv F_{p}\left(h_{i}, p_{i}\right)
\end{aligned}
$$

Here $\tau=a t, \alpha=b / K, \eta=m / a, \epsilon=a / r$ and we have rescaled the populations according to $h_{i} \rightarrow h_{i} / b, p_{i} \rightarrow(a c / r b) p_{i}$. The pair of equations (2) can be rewritten in the vector form

$$
\frac{d \mathbf{x}_{i}}{d t}=\mathbf{F}\left(\mathbf{x}_{i}\right)
$$

where $\mathbf{x}_{i}=\left(h_{i}, p_{i}\right)^{T}$ and $\mathbf{F}=\left(F_{h}, F_{p}\right)^{T}$. It has previously been shown that $\alpha<1$ and $\eta<(1-\alpha) /(1+\alpha)$ is a parameter regime where stable limit cycles exist [18]. Two examples of deterministic limit cycles are shown in Figure 1.

Migration of the predator and prey species between patches is represented by the per capita rates $D_{h}$ and $D_{p}$ and the symmetric connectivity matrix $C_{i j}$. 
The component $C_{i j}=1$ if there is mutual migration between the $i$-th and $j$-th patch, and $C_{i j}=0$ otherwise. The Moran effect is modeled by assuming that all of the predator populations and all of the prey populations are driven by common white noise terms $\eta_{p}(t)$ and $\eta_{h}(t)$, respectively. That is, $\eta_{p}(t)$ and $\eta_{p}(t)$ are described by zero mean Gaussian processes with two-point correlations

$$
\begin{aligned}
\left\langle\eta_{p}(t) \eta_{p}\left(t^{\prime}\right)\right\rangle & =\delta\left(t-t^{\prime}\right), \\
\left\langle\eta_{h}(t) \eta_{h}\left(t^{\prime}\right)\right\rangle & =\delta\left(t-t^{\prime}\right), \\
\left\langle\eta_{p}(t) \eta_{h}\left(t^{\prime}\right)\right\rangle & =0
\end{aligned}
$$

where $\delta(t)$ denotes the Dirac delta function. From now on we set $D_{h}=D_{p}=$ $D$ with $D$ a small parameter in order to simplify the analysis. Also, taking the strength of the extrinsic noise to be scaled by a small parameter $\sigma$, we obtain the following system of Langevin equations for the stochastic population variables $\mathbf{X}_{i}=\left(H_{i}, P_{i}\right)^{T}$ :

$$
\begin{aligned}
\frac{d H_{i}}{d \tau} & =F_{h}\left(\mathbf{X}_{i}\right)+D \sum_{j=1}^{N} C_{i j}\left(H_{j}-H_{i}\right)+\sigma \eta_{h}(t) \\
\frac{d P_{i}}{d \tau} & =F_{p}\left(\mathbf{X}_{i}\right)+D \sum_{j=1}^{N} C_{i j}\left(P_{j}-P_{i}\right)+\sigma \eta_{p}(t)
\end{aligned}
$$

\subsection{Phase Reduction and Averaging}

Suppose that in the absence of dispersal and external noise, each patch acts dynamically as a limit cycle oscillator. Following previous studies of noise-induced phase synchronization $[31,37,10,38,22,20]$, we introduce a single scalar variable $\theta_{i} \in[0,2 \pi)$ that represents the phase of the $i$-th oscillator. That is, each isolated limit cycle evolves according to the simple phase equation $\dot{\theta}_{i}=\omega$, where $\omega$ is the natural frequency of the oscillator and the phase-plane representation of the limit cycle is $\mathbf{x}_{i}^{*}(t)=\mathbf{x}^{*}\left(\theta_{i}(t)\right)$. The notion of phase is then extended into some neighborhood $\mathcal{M} \in \mathbb{R}^{2}$ of the $i$-th limit cycle using an isochronal mapping $\Pi: \mathcal{M} \rightarrow[0,2 \pi)$ with $\theta_{i}=\Pi\left(\mathbf{x}_{i}\right)$. Assuming that the limit cycle is sufficiently attracting in the presence of weak dispersal and noise, the dynamics can be restricted to the neighborhood $\mathcal{M}$ with high probability. This allows us to define a stochastic phase variable for each oscillator according to $\Theta_{i}=\Pi\left(\mathbf{X}_{i}\right)$ with $\mathbf{X}_{i}$ evolving according to the Langevin equation (5). Moreover, the standard deterministic phase reduction method used in $[11,12]$ can be extended to the Langevin equation (5), provided that the noise is treated in the sense of Stratonovich [9]. That is, the corresponding Wiener processes $d W_{h}(t)=\eta_{h}(t) d t$ and $d W_{p}(t)=\eta_{p}(t) d t$ are considered the zero correlation limits of a coloured noise process so that the normal rules of calculus can be applied. Carrying out the phase reduction then leads to the following stochastic differential equation (SDE) for the stochastic phase 
variables $\Theta_{i}(t)[37,29,38]$ :

$$
d \Theta_{i}=\left(\omega+D \mathbf{Z}\left(\Theta_{i}\right) \cdot \sum_{j=1}^{N} C_{i j}\left(\begin{array}{c}
H\left(\Theta_{j}\right)-H\left(\Theta_{i}\right) \\
P\left(\Theta_{j}\right)-P\left(\Theta_{i}\right)
\end{array}\right)\right) d t+\sigma \mathbf{Z}\left(\Theta_{i}\right) \cdot d \mathbf{W}(t)
$$

where $d \mathbf{W}(t)=\left(d W_{h}, d W_{p}\right)^{T}$ such that $\left\langle d W_{k}(t)\right\rangle=0$ and

$$
\left\langle d W_{k}(t) d W_{l}(t)\right\rangle=\delta_{k, l} d t .
$$

Here $\mathbf{Z}=\left(Z_{h}, Z_{p}\right)$ is the infinitesimal phase resetting curve (PRC) whose components are defined according to

$$
\left.Z_{k}(\theta) \equiv \frac{\partial}{\partial x_{k}} \Pi\right|_{\mathbf{x}=\mathbf{x}^{*}(\theta)}, \quad k=h, p
$$

such that $\sum_{k=1,2} Z_{k}(\theta) F_{k}\left(\mathbf{x}^{*}(\theta)\right)=\omega$. Note that all terms multiplying the $\mathrm{PRC}$ in equation (6) are evaluated on the limit cycle so that, for example, $H\left(\Theta_{j}\right)=H_{j}\left(\mathbf{x}^{*}\left(\Theta_{j}\right)\right)$. It can be shown that the PRC is the unique $2 \pi$-periodic solution of the adjoint linear equation [4]

$$
\frac{d Z_{k}}{d t}=-\sum_{j=h, p} F_{j k}\left(\mathbf{x}^{*}(t)\right) Z_{j}(t)
$$

where $F_{j k}=\partial F_{j} / \partial x_{k}$. The PRC can be evaluated numerically by solving the adjoint equation backwards in time, since all non-zero Floquet exponents of solutions to the adjoint equation have positive real part.

In order to simplify the analysis of noise-induced synchronization, we now convert equation (6) from a Stratonovich to an Ito system of Langevin equations:

$$
\begin{aligned}
d \Theta_{i}= & {\left[\omega d t+\frac{\sigma^{2}}{2} \mathbf{Z}\left(\Theta_{i}\right) \cdot \partial_{\Theta_{i}} \mathbf{Z}\left(\Theta_{i}\right)\right] d t } \\
& +D \mathbf{Z}\left(\Theta_{i}\right) \cdot \sum_{j=1}^{N} C_{i j} \mathbf{P}\left(\Theta_{i}, \Theta_{j}\right) d t+\sigma \mathbf{Z}\left(\theta_{i}\right) \cdot d \mathbf{W}(t),
\end{aligned}
$$

where we have set

$$
\left(\begin{array}{c}
H\left(\Theta_{j}\right)-H\left(\Theta_{i}\right) \\
P\left(\Theta_{j}\right)-P\left(\Theta_{i}\right)
\end{array}\right):=\mathbf{P}\left(\Theta_{i}, \Theta_{j}\right)
$$

The $\mathcal{O}(\sigma)$ correction to the natural frequency is a consequence of the conversion from Stratonovich to Ito noise [9]. Equation (10) can be rewritten in the more compact form

$$
d \Theta_{i}=\mathcal{A}_{i}(\boldsymbol{\Theta}) d t+d \zeta_{i}(\boldsymbol{\Theta}, t)
$$

where $\left\{\zeta_{i}(\boldsymbol{\Theta}, t)\right\}$ are correlated Wiener processes and $\boldsymbol{\Theta}=\left(\Theta_{1}, \ldots, \Theta_{N}\right)$. That is,

$$
d \zeta_{i}(\boldsymbol{\Theta}, t)=\sigma \mathbf{Z}\left(\Theta_{i}\right) \cdot d \mathbf{W}(t)
$$


with

$$
\left\langle d \zeta_{i}(\boldsymbol{\Theta}, t)\right\rangle=0, \quad\left\langle d \zeta_{i}(\boldsymbol{\Theta}, t) d \zeta_{j}(\boldsymbol{\Theta}, t)\right\rangle=\Delta_{i j}(\boldsymbol{\Theta}) d t
$$

Here $\Delta_{i j}(\boldsymbol{\Theta})$ is the equal-time correlation matrix

$$
\Delta_{i j}(\boldsymbol{\Theta})=\sigma^{2} \sum_{k=h, p}^{M} Z_{k}\left(\Theta_{i}\right) Z_{k}\left(\Theta_{j}\right)
$$

The drift term $\mathcal{A}_{i}(\boldsymbol{\Theta})$ is given by

$$
\mathcal{A}_{i}(\boldsymbol{\Theta})=\omega+\frac{\sigma^{2}}{2} \mathbf{Z}\left(\Theta_{i}\right) \cdot \partial_{\Theta_{i}} \mathbf{Z}\left(\Theta_{i}\right)+D \mathbf{Z}\left(\Theta_{i}\right) \cdot \sum_{j=1}^{N} C_{i j} \mathbf{P}\left(\Theta_{i}, \Theta_{j}\right)
$$

It follows that the ensemble is described by a multivariate Fokker-Planck equation of the form

$$
\frac{\partial P(\boldsymbol{\theta}, t)}{\partial t}=-\sum_{i=1}^{N} \frac{\partial}{\partial \theta_{i}}\left[\mathcal{A}_{i}(\boldsymbol{\theta}) P(\boldsymbol{\theta}, t)\right]+\frac{1}{2} \sum_{i, j=1}^{N} \frac{\partial^{2}}{\partial \theta_{i} \partial \theta_{j}}\left[\Delta_{i j}(\boldsymbol{\theta}) P(\boldsymbol{\theta}, t)\right]
$$

As first shown in Ref. [41], and further developed in Ref. [38], considerable care must be taken in carrying out the phase reduction procedure in the presence of Gaussian white noise in order to obtain the correct form of the drift terms $\mathcal{A}_{i}(\boldsymbol{\Theta})$. Since we are assuming that the external white noise is Stratonovich (ie. the limit of a colored noise process), the correction terms derived in [38] are not required provided that the limit cycles are sufficiently strongly attracting. Even if this is not the case, neglecting such terms does not affect our subsequent analysis.

Having obtained the FP equation (16), we can now carry out the averaging procedure of Nakao et. al. [29], see also [22]. The basic idea is to introduce the slow phase variables $\boldsymbol{\psi}=\left(\psi_{1}, \ldots, \psi_{N}\right)$ according to $\theta_{i}=\omega t+\psi_{i}$ and set $Q(\boldsymbol{\psi}, t)=P\left(\left\{\omega t+\theta_{i}\right\}, t\right)$. For sufficiently small $D$ and $\sigma, Q$ is a slowly varying function of time so that we can average the Fokker-Planck equation for $Q$ over one cycle of length $T=2 \pi / \omega$. The averaged FP equation for $Q$ is thus [29]

$$
\frac{\partial Q(\boldsymbol{\psi}, t)}{\partial t}=-\sum_{i=1}^{N} \frac{\partial}{\partial \psi_{i}}\left[\overline{\mathcal{A}}_{i}(\boldsymbol{\psi}) Q(\boldsymbol{\psi}, t)\right]+\frac{1}{2} \sum_{i, j=1}^{\mathcal{N}} \frac{\partial^{2}}{\partial \psi_{i} \partial \psi_{j}}\left[\bar{\Delta}_{i j}(\boldsymbol{\psi}) Q(\boldsymbol{\psi}, t)\right]
$$


where

$$
\begin{aligned}
\overline{\mathcal{A}}_{i}= & \frac{1}{T} \int_{0}^{T} \mathcal{A}_{j}\left(\omega t+\psi_{1}, \ldots, \omega t+\psi_{N}\right) d t-\omega, \\
= & \frac{D}{T} \int_{0}^{T} \mathbf{Z}\left(\omega t+\psi_{i}\right) \cdot \sum_{j=1}^{N} C_{i j} \mathbf{P}\left(\omega t+\psi_{i}, \omega t+\psi_{j}\right) d t \\
& +\frac{\sigma^{2}}{2 T} \int_{0}^{T} \mathbf{Z}\left(\Theta_{i}\right) \cdot \partial_{\Theta_{i}} \mathbf{Z}\left(\Theta_{i}\right) d t \\
= & D \sum_{j=1}^{N} C_{i j} H\left(\psi_{j}-\psi_{i}\right),
\end{aligned}
$$

since $\left[\mathbf{Z}\left(\Theta_{i}\right)^{2}\right]_{0}^{T}=0$, and

$$
\begin{aligned}
\bar{\Delta}_{i j}(\boldsymbol{\psi}) & =\frac{1}{T} \int_{0}^{T} \Delta_{i j}\left(\omega t+\psi_{1}, \ldots, \omega t+\psi_{N}\right) d t \\
& =\frac{\sigma^{2}}{T} \int_{0}^{T} \sum_{k=h, p}^{M}\left[Z_{k}\left(\omega t+\psi_{i}\right) Z_{k}\left(\omega t+\psi_{j}\right)\right] d t \\
& =\sigma^{2} \Delta\left(\psi_{j}-\psi_{i}\right)
\end{aligned}
$$

with

$$
H(\psi)=\frac{1}{T} \int_{0}^{T} \mathbf{Z}(\omega t) \cdot \mathbf{P}(\omega t, \omega t+\psi) d t
$$

and

$$
\Delta(\psi)=\frac{1}{T} \int_{0}^{T} \sum_{k=h, p} Z_{k}(\omega t) Z_{k}(\omega t+\psi) d t .
$$

The averaged FP equation has a corresponding SDE that describes the evolution of the stochastic phases $\Psi_{i}(t)$ :

$$
d \Psi_{i}=D \sum_{j=1}^{N} C_{i j} H\left(\Psi_{j}-\Psi_{i}\right) d t+d \bar{\zeta}_{i}(\boldsymbol{\Psi}, t)
$$

with

$$
\left\langle d \bar{\zeta}_{i}(\boldsymbol{\Psi}, t)\right\rangle=0, \quad\left\langle d \bar{\zeta}_{i}(\boldsymbol{\Psi}, t) d \bar{\zeta}_{j}(\boldsymbol{\Psi}, t)\right\rangle=\bar{\Delta}_{i j}(\boldsymbol{\Psi}) d t
$$

2.3 Results for a pair of oscillators

Let us now focus on a pair of oscillators $(N=2)$ with $C_{12}=C_{21}=1$, and define the phase difference $\Phi=\Psi_{2}-\Psi_{1}$. The averaged SDE (23) reduces to the pair of equations

$$
\begin{aligned}
& d \Psi_{1}=D H(\Phi) d t+d \bar{\zeta}_{1}(\Phi, t) \\
& d \Psi_{2}=D H(-\Phi) d t+d \bar{\zeta}_{2}(\Phi, t)
\end{aligned}
$$



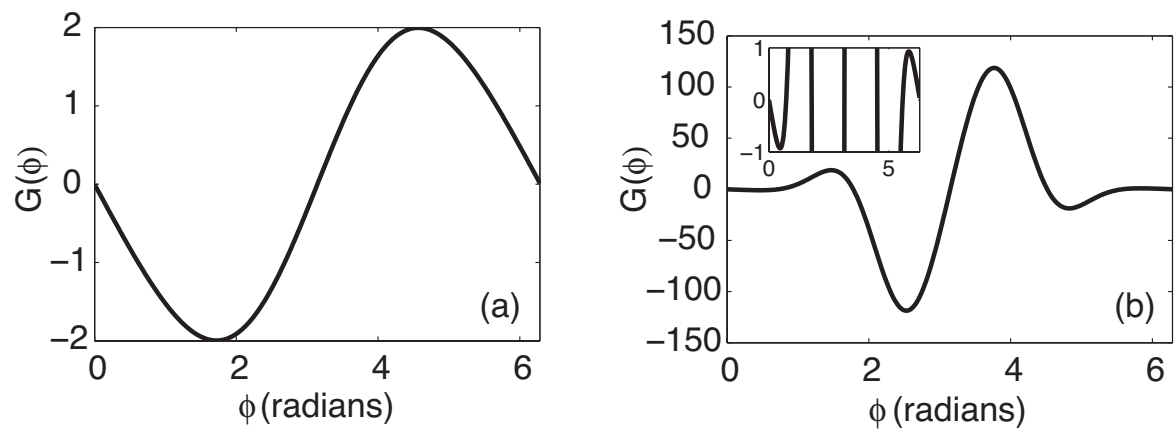

Fig. 2 Numerically evaluated G-functions. (a) $\epsilon=0.1, \alpha=0.4, \eta=0.4$. Only stable phaselocked state is the synchronous state $\phi=0$. (b) $\epsilon=0.1, \alpha=0.3, \eta=0.3$. There is now an additional pair of stable asynchronous states. Inset: Close-up of behaviour near $\phi=0$.

with

$$
\begin{aligned}
& \left\langle d \bar{\zeta}_{1}(\Phi, t) d \bar{\zeta}_{1}(\Phi, t)\right\rangle=\left\langle d \bar{\zeta}_{2}(\Phi, t) d \bar{\zeta}_{2}(\Phi, t)\right\rangle=\sigma^{2} \Delta(0) \\
& \left\langle d \bar{\zeta}_{1}(\Phi, t) d \bar{\zeta}_{2}(\Phi, t)\right\rangle=\left\langle d \bar{\zeta}_{2}(\Phi, t) d \bar{\zeta}_{1}(\Phi, t)\right\rangle=\sigma^{2} \Delta(\Phi)
\end{aligned}
$$

Taking the difference of the equations (25) and using the result that the difference between two Gaussian random variables is also Gaussian leads to the scalar SDE

$$
d \Phi=D G(\Phi) d t+\sigma K(\Phi) d W(t)
$$

where $G(\phi)=H(\phi)-H(-\phi)$ is the odd part of the phase interaction function $H, W(t)$ is a Wiener process and

$$
\begin{aligned}
K(\Phi) & =\frac{1}{\sigma} \sqrt{\left\langle\left[d \bar{\zeta}_{2}(\Phi, t)-d \bar{\zeta}_{1}(\Phi, t)\right]\left[d \bar{\zeta}_{2}(\Phi, t)-d \bar{\zeta}_{1}(\Phi, t)\right]\right\rangle} \\
& =\sqrt{2[\Delta(0)-\Delta(\Phi)]}
\end{aligned}
$$

In the absence of extrinsic noise, the dynamics of the phase difference $\phi$ is given by the deterministic differential equation $[4,11]$

$$
\frac{d \phi}{d t}=D G(\phi)
$$

Conditions for synchrony are then determined entirely by the function $G$, which itself depends on the PRC and the nature of the diffusive coupling. The steady or phase-locked states are given by the zeros of $G, G(\phi)=0$, and are stable when $G^{\prime}(\phi)<0$ and unstable when $G^{\prime}(\phi)>0$. The rate of convergence to a given steady state is determined by the magnitude of $G^{\prime}(\phi)$ at that point. In Fig. 2, we plot $G(\phi)$ for the same two sets of parameters used in Fig. 1. In Fig. 2(a) the synchronous state $\phi=0$ is the only stable steady state, whereas in Fig. 2(b) there are additional stable asynchronous states at $\phi \approx 1.86$ (and by symmetry $\phi \approx 2 \pi-1.86$ ); the asynchronous states have a much larger basin of attraction compared to the synchronous state. When 

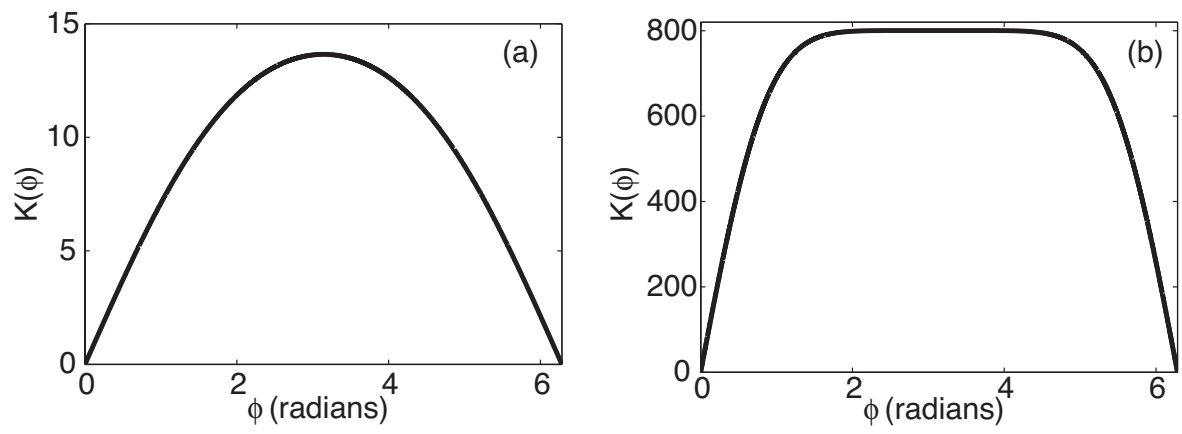

Fig. 3 Numerically evaluated K-functions. Left: (a) $\epsilon=0.1, \alpha=0.4, \eta=0.4$. (b) $\epsilon=0.1$, $\alpha=0.3, \eta=0.3$.

noise is included, the stochastic phase difference evolves according to the SDE (27) with corresponding FP equation of the form

$$
\frac{\partial P(\phi, t)}{\partial t}=-D \frac{\partial}{\partial \phi} G(\phi) P(\phi, t)+\frac{\sigma^{2}}{2} \frac{\partial^{2}}{\partial \phi^{2}} K^{2}(\phi) P(\phi, t)
$$

The steady-state solution $P_{0}$ satisfies the equation

$$
G(\phi) P_{0}(\phi)=\frac{\sigma^{2}}{2 D} \frac{d}{d \phi} K^{2}(\phi) P_{0}(\phi)
$$

which implies that

$$
K^{2}(\phi) P_{0}(\phi)=A \exp \left(\int_{0}^{\phi} \frac{2 D G(\theta)}{\sigma^{2} K^{2}(\theta)} d \theta\right)
$$

Figure 3 shows that the K-function has zeros at $\phi=0=2 \pi$, suggesting that the steady-state PDF $P_{0}(\phi)$ blows up at $\phi=0$, that is, $P(\phi)=\delta(\phi)$. This implies that the common noise source causes total synchronization in the limit $t \rightarrow \infty$.

In order to look at transient behavior, we solve the FP equation (30) numerically assuming the initial condition $P(\phi, 0)=\delta\left(\phi-\phi_{0}\right)$. The results are shown in Fig 4(a) for the first parameter set of Figs. 1-3, for which there are no asynchronous phase-locked states. The initial delta function at $\phi=\phi_{0} \neq 0$ first broadens as it moves towards the steady state at $\phi=0$, and then narrows again as it tends towards a delta function centered at $\phi=0$, indicating complete phase synchronization. In Fig. 4(b) we plot the time evolution of one measure of the degree of synchrony $\Pi_{0}(t)$, namely, $\Pi_{0}(t)=\int_{-\Delta}^{\Delta} p(\phi, t) d \phi$. That is, $\Pi_{0}(t)$ is the probability that at time $t$ the phase difference $\phi$ is within $\Delta$ radians of the synchronous state $\phi=0$. For concreteness we take $\Delta=0.2$, although the results do not depend on the precise choice for $\Delta$ provided $\Delta \ll \pi$. It can be seen that there is a long latency before synchrony begins to develop, reflecting the fact that the PDF at a each time step is well approximated by 

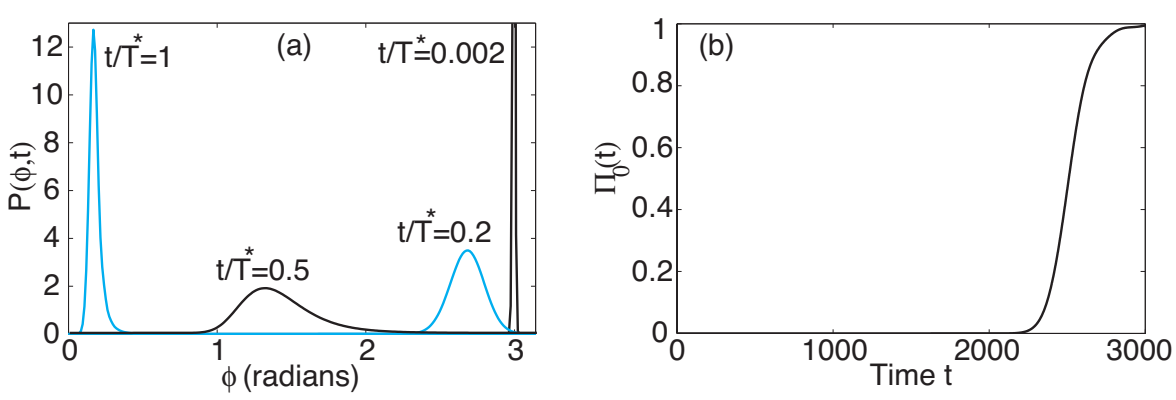

Fig. 4 Monostable case: $\epsilon=0.1, \alpha=0.4, \eta=0.4$ with dispersal coefficient $D=0.001$ and $\sigma=2 \times 10^{-4}$. The initial condition is a delta function $\delta\left(\phi-\phi_{0}\right)$ with $\phi_{0}=3$ radians. (a) The $\operatorname{PDF} P(\phi, t)$ is plotted as a function of $\phi$ at several points in time $t$, normalised with respect to the time $T^{*}$ taken to synchrony. (The peak at $t / T^{*}=0.002$ is not shown here as it would distort the scale of the figure.) Synchrony here is defined as when $\Pi_{0}(t)=\int_{-0.2}^{0.2} p(\phi, t) d \phi>$ 0.75 , which yields $T^{*}=2589$ or around 500 cycles. (b) Plot of $\Pi_{0}(t)$ against time.
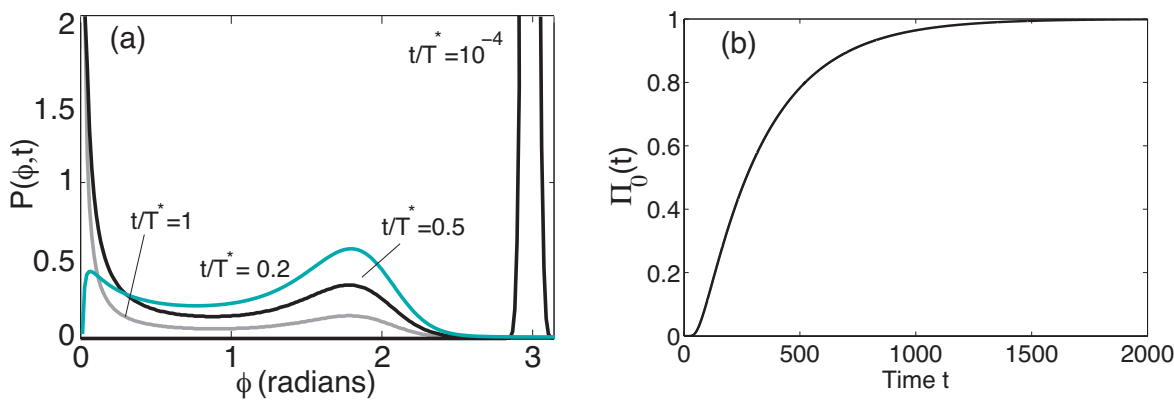

Fig. 5 Multistable case: $\epsilon=0.1, \alpha=0.3, \eta=0.3$ with dispersal $D=0.001$ and $\sigma=$ $2 \times 10^{-4}$. The initial condition is a delta function $\delta\left(\phi-\phi_{0}\right)$ with $\phi_{0}=3$ radians. (a) The PDF $P(\phi, t)$ is plotted as a function of $\phi$ at several points in time $t$ normalised with respect to the time $T^{*}$ taken to synchrony. Synchrony is defined as in Fig. 4 but now $T^{*}=461$. (b) Plot of $\Pi_{0}(t)$ against time.

a localized Gaussian. For the second parameter set of Figs. 1-3, for which the synchronous state coexists with a pair of stable asynchronous states, the temporal evolution of the PDF is very different. For example, if the initial phase is within the basin of attraction of the asynchronous state $\phi=\phi_{a} \approx 1.86$ in the absence of noise, then the corresponding PDF develops a bimodal structure at intermediate times before moving towards $\phi=0$ and eventually converging to the expected delta function, see Fig. 5(a). Hence, the time-dependent PDF is much broader than the previous case. Note, however, that the approach to synchrony is now much faster, see Fig. 5(b). This is due to the fact that the synchronization rate depends on the scale of the phase interaction function $G(\phi)$, which is much larger for the second scenario, see Fig. 2. In Fig. 6(a) we directly compare the two cases by determining how the time to synchrony $T$ varies with the initial phase $\phi_{0}$. In order to make the comparison, we normalize time with respect to the total time to synchrony $T^{*}$. In the absence of an asyn- 

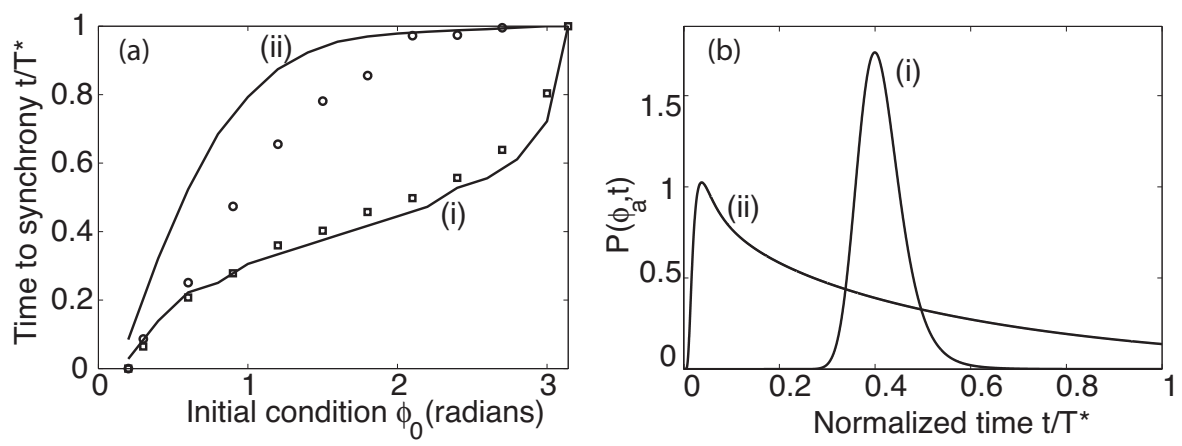

Fig. 6 Comparison of (i) monostable and (ii) multistable cases. (a) Plots of time taken to synchrony $T$ (normalized with respect to $T^{*}=T\left(\phi_{0}=3\right)$ ) as a function of the initial condition $\phi_{0}$. Continuous curves are based on solutions to the FP equation (30). Data points are based on numerical solutions of the full system of RM equations (5). (b) Plot of the PDF $P(\phi, t)$ at the asynchronous state $\phi_{a} \approx 1.86$.

chronous state, $T$ varies approximately linearly with $\phi_{0}$. On the other hand, $T$ is a step-like function of $\phi_{0}$ in the presence of the asynchronous state $\phi_{a}$. The presence or absence of an asynchronous state can also be discerned by plotting the probability density $P\left(\phi_{a}, t\right)$ as a function of time, see Fig. 6(b). In Fig. $6(\mathrm{a})$ we also show data points based on direct numerical simulations of the full system of RM equations (5). It can be seen that there is excellent agreement between the full model and the reduced phase model in the monostable case. The reduced model is less accurate in the multistable case but the basic trend is still captured. Note that one source of error is that the criterion for synchrony in the reduced model and the full model are different. In the former case, we define the time to synchrony to be when $\Pi_{0}(t)=\int_{-0.2}^{0.2} p(\phi, t) d \phi>0.75$. On the other hand, in simulations of the full model equations we first calculated the deterministic limit cycle by solving the R-M equations (2), and used this to generate an initial condition with phase difference $\phi_{0}$. We then ran the full stochastic equations (5) forward in time until the root-mean-squared distance between the two oscillators (in the $\mathrm{H}-\mathrm{P}$ plane) was less than $10^{-8}$. This criterion for synchrony allowed us to avoid having to determine the phase of each oscillator at each time-step in time, which would have been computationally intensive and potentially inaccurate due to the highly curved isochrons.

\section{Introducing uncorrelated noise}

So far we have considered the effects of a common or fully correlated extrinsic noise source (environmental noise) on the synchronization of a pair of predatorprey oscillators. However, a basic assumption of the above model is that in the absence of environmental noise, each predator-prey patch can be modeled by a deterministic system of ODEs in which the number of predators and prey are treated as continuous variables. Such an approximation is reasonable when 
the populations within a patch are sufficiently large. In recent years there has been a growing interest in developing stochastic individual-based descriptions of ecological systems in which explicit rules determining the interaction of individuals are specified $[34,27,7,8]$. Under the assumption that the system is well mixed, one can derive deterministic ODE models in the mean-field limit. One of the advantages of individual-based models is that it is also possible to study the effects of demographic noise for finite populations (finite-size effects). One example of such a theory has been developed by McKane and Newman based on a stochastic urn model [23,24]. In this type of model a single patch is divided into $M$ plots of equal area, with the plot size chosen so that each one contains one prey (species A) or one predator (species B) or neither (an empty plot denoted by E). One then imagines taking all the A, B and E from their particular sites and mixing them together into a single patch in which spatial location is now ignored. The time evolution of the model is then specified as follows: at each time step randomly select either a single individual or a pair of individuals from the population and implement a set of rules associated with the given model. These rules take the form of state transitions with modeldependent transition rates. For example, $A \rightarrow E$ and $A E \rightarrow A A$ represent the death and birth of an individual of species $A$, respectively, whereas $A B \rightarrow E B$ represents a predator-prey interaction.

Since a stochastic urn model realises a Markov process, it is possible to describe a continuous time version of the model in terms of a master equation $[23,24,20]$. The latter describes the evolution of the probability $P\left(m_{A}, m_{B}, t\right)$ that there are $M_{A}(t)=m_{A}$ individuals of species $A$ and $M_{B}(t)=m_{B}$ individuals of species $B$ within a patch at time $t$, with the constraint $M_{A}(t)+$ $M_{B}(t)+M_{E}(t)=M$. For large but finite $M$ one can then carry out a systemsize expansion familiar from the study of chemical master equations [39], in order to approximate the stochastic dynamics in terms of an FP equation. The latter describes the evolution of the PDF of an associated SDE for the stochastic variables $X_{A}(t)=M_{A}(t) / M$ and $X_{B}(t)=M_{B}(t) / M$ with $X_{A}, X_{B}$ treated as continuous variables. The main consequence of such an analysis is that finite size effects generate a source of demographic noise. Of particular significance for our present model is that demographic noise is uncorrelated across different patches within a metacommunity. Previously, we have used a stochastic urn model to analyse the combined effects of correlated environmental noise (Moran effect) and uncorrelated demographic noise on the stochastic synchronisation of a population of uncoupled predator-prey oscillators [20]. As one might expect, the inclusion of uncorrelated noise counteracts the synchronising effects of environmental noise with the degree of synchronization an increasing function of $M$. However, as we show below, the effects of demographic noise are more subtle when dispersal is also taken into account.

Rather than deriving the SDE for a system of coupled RM oscillators with dispersal, environmental noise and demographic noise, starting from a corresponding stochastic urn model, here we simply treat uncorrelated noise as an 
extra additive noise term in the Langevin equations (5):

$$
\begin{aligned}
\frac{d H_{i}}{d \tau} & =F_{h}\left(\mathbf{X}_{i}\right)+D \sum_{j=1}^{N} C_{i j}\left(H_{j}-H_{i}\right)+\sigma \eta_{h}(t)+\rho \sigma \eta_{h, i}(t) \\
\frac{d P_{i}}{d \tau} & =F_{p}\left(\mathbf{X}_{i}\right)+D \sum_{j=1}^{N} C_{i j}\left(P_{j}-P_{i}\right)+\sigma \eta_{p}(t)+\rho \sigma \eta_{p, i}(t)
\end{aligned}
$$

where each $\eta_{h, i}$ and $\eta_{p, i}$ are independent white noise processes. Thus for $r=$ $p, h, s=p, h$ and $i=1, \ldots, N$

$$
\begin{aligned}
\left\langle\eta_{r, i}(t) \eta_{s, j}\left(t^{\prime}\right)\right\rangle & =\delta\left(t-t^{\prime}\right) \delta_{r, s} \delta_{i, j}, \\
\left\langle\eta_{r, i}(t) \eta_{s}\left(t^{\prime}\right)\right\rangle & =0 .
\end{aligned}
$$

The above simplification is also motivated by the fact that the environment itself could be a source of uncorrelated noise, that is, environmental effects such as the weather need not be perfectly correlated across different patches. Note that an analogous system of equations with a mixture of correlated and uncorrelated noise has been studied at some length by Ly and Ermentrout [22] within the context of a pair of coupled neuronal oscillators. However, these authors were mainly concerned with developing a computationally efficient method for calculating the steady-state PDF based on the use of Fourier methods and asymptotic reduction techniques. They then applied their phase reduction method to study the behavior of a realistic synaptically-coupled system of Morris-Lecar oscillators. One of their main results was to show that the combination of weak coupling and partially correlated noise can lead to bistability (in the probabilistic sense) between a synchronous and an asynchronous state. An analogous result will hold in the case of our population model.

We now proceed along identical lines to section 2. That is, applying the phase reduction method ultimately leads to the following Ito SDE for the stochastic phases:

$$
d \Theta_{i}=\mathcal{A}_{i}^{*}(\boldsymbol{\Theta}) d t+d \xi_{i}(\boldsymbol{\Theta}, t),
$$

where

$$
\mathcal{A}_{i}^{*}(\boldsymbol{\Theta})=\omega+\frac{\left(1+\rho^{2}\right) \sigma^{2}}{2} \mathbf{Z}\left(\Theta_{i}\right) \cdot \partial_{\Theta_{i}} \mathbf{Z}\left(\Theta_{i}\right)+D \mathbf{Z}\left(\Theta_{i}\right) \cdot \sum_{j=1}^{N} C_{i j} \mathbf{P}\left(\Theta_{i}, \Theta_{j}\right),
$$

and $d \xi_{i}(\boldsymbol{\Theta}, t)=\sigma \mathbf{Z}\left(\Theta_{i}\right) \cdot\left(d \mathbf{W}(t)+\rho d \mathbf{W}_{i}(t)\right)$ are correlated Wiener processes, so that

$$
\left\langle d \xi_{i}(\boldsymbol{\Theta}, t)\right\rangle=0, \quad\left\langle d \xi_{i}(\boldsymbol{\Theta}, t) d \xi_{j}(\boldsymbol{\Theta}, t)\right\rangle=\Delta_{i j}^{*}(\boldsymbol{\Theta}) d t
$$

where the equal-time correlation matrix $\Delta_{i j}^{*}(\boldsymbol{\Theta})$ is now

$$
\Delta_{i j}^{*}(\boldsymbol{\Theta})=\sigma^{2}\left(1+\rho^{2} \delta_{i, j}\right) \sum_{k=h, p}^{M} Z_{k}\left(\Theta_{i}\right) Z_{k}\left(\Theta_{j}\right) .
$$


It follows that the ensemble is described by the multivariate Fokker-Planck equation (16) with $\mathcal{A}_{i}(\boldsymbol{\Theta}) \rightarrow \mathcal{A}_{i}^{*}(\boldsymbol{\Theta})$ and $\Delta_{i j}(\boldsymbol{\Theta}) \rightarrow \Delta_{i j}^{*}(\boldsymbol{\Theta})$. Averaging over a single period as before, generates the averaged $\mathrm{FP}$ equation

$$
\frac{\partial Q^{*}(\boldsymbol{\psi}, t)}{\partial t}=-\sum_{i=1}^{N} \frac{\partial}{\partial \psi_{i}}\left[\overline{\mathcal{A}_{i}^{*}}(\boldsymbol{\psi}) Q^{*}(\boldsymbol{\psi}, t)\right]+\frac{1}{2} \sum_{i, j=1}^{\mathcal{N}} \frac{\partial^{2}}{\partial \psi_{i} \partial \psi_{j}}\left[\overline{\Delta_{i j}^{*}}(\boldsymbol{\psi}) Q^{*}(\boldsymbol{\psi}, t)\right]
$$

where

$$
\overline{\mathcal{A}_{i}^{*}}=\overline{\mathcal{A}_{i}} \equiv D \sum_{j=1}^{N} C_{i j} H\left(\psi_{j}-\psi_{i}\right)
$$

and

$$
\begin{aligned}
\overline{\Delta_{i j}^{*}} & =\left(1+\rho^{2} \delta_{i, j}\right) \overline{\Delta_{i j}} \\
& =\left(1+\rho^{2} \delta_{i, j}\right) \sigma^{2} \Delta\left(\psi_{j}-\psi_{i}\right) \\
& =\sigma^{2}\left(\Delta\left(\psi_{j}-\psi_{i}\right)+\rho^{2} \Delta(0)\right)
\end{aligned}
$$

with $H$ and $\Delta$ defined according to equations (21) and (22).

As in section 2, let us now focus on a pair of oscillators symmetrically coupled $\left(C_{12}=C_{21}=1\right)$, and define the phase difference $\phi=\psi_{2}-\psi_{1}$. The SDE corresponding to the Fokker-Planck equation (40) for $N=2$ takes the form

$$
\begin{aligned}
& d \Psi_{1}=D H(\Phi) d t+d \bar{\xi}_{1}(\Phi, t) \\
& d \Psi_{2}=D H(-\Phi) d t+d \bar{\xi}_{2}(\Phi, t)
\end{aligned}
$$

with

$$
\begin{aligned}
& \left\langle d \bar{\xi}_{1}(\Phi, t) d \bar{\xi}_{1}(\Phi, t)\right\rangle=\left\langle d \bar{\xi}_{2}(\Phi, t) d \bar{\xi}_{2}(\Phi, t)\right\rangle=\sigma^{2}\left(1+\rho^{2}\right) \Delta(0) \\
& \left\langle d \bar{\xi}_{1}(\Phi, t) d \bar{\xi}_{2}(\Phi, t)\right\rangle=\left\langle d \bar{\xi}_{2}(\Phi, t) d \bar{\xi}_{1}(\Phi, t)\right\rangle=\sigma^{2} \Delta(\Phi)
\end{aligned}
$$

Taking the difference of the two equations yields the SDE

$$
d \Phi=D G(\Phi) d t+\sigma K^{*}(\Phi) d W(t)
$$

where $G(\phi)=H(\phi)-H(-\phi)$ is the phase-interaction function of section 2 , and

$$
\begin{aligned}
K^{*}(\Phi) & =\frac{1}{\sigma} \sqrt{\left\langle\left[d \bar{\xi}_{2}(\Phi, t)-d \bar{\xi}_{1}(\Phi, t)\right]\left[d \bar{\xi}_{2}(\Phi, t)-d \bar{\xi}_{1}(\Phi, t)\right]\right\rangle} \\
& =\sqrt{2\left[\left(1+\rho^{2}\right) \Delta(0)-\Delta(\Phi)\right]} .
\end{aligned}
$$

Equation (43) has an associated PDF that evolves according to the FP equation

$$
\frac{\partial P}{\partial t}=-\frac{\partial}{\partial \Phi} G P+\frac{\sigma^{2}}{2} \frac{\partial^{2}}{\partial \Phi^{2}}\left[K^{* 2} P\right]
$$


Solving for the steady state density $P_{0}$ gives

$$
G P_{0}=\frac{\sigma^{2}}{2} \frac{d}{d \phi}\left[K^{* 2} P_{0}\right]
$$

This implies that

$$
K^{* 2}(\Phi) P_{0}(\Phi)=A \exp \left(\int_{0}^{\phi} \frac{2 D G(\theta)}{\sigma^{2} K^{* 2}(\theta)} d \theta\right)
$$

with

$$
P_{0}(\Phi)=\frac{A}{2\left(1+\rho^{2}\right) \Delta(0)-\Delta(\Phi)} \exp \left(\int_{0}^{\phi} \frac{D G(\theta) d \theta}{\sigma^{2}\left[\left(1+\rho^{2}\right) \Delta(0)-\Delta(\theta)\right.}\right)
$$

Here $A$ is a constant of integration, which can be determined from the normalization condition

$$
\int_{0}^{2 \pi} P_{0}(\Phi) d \Phi=1
$$
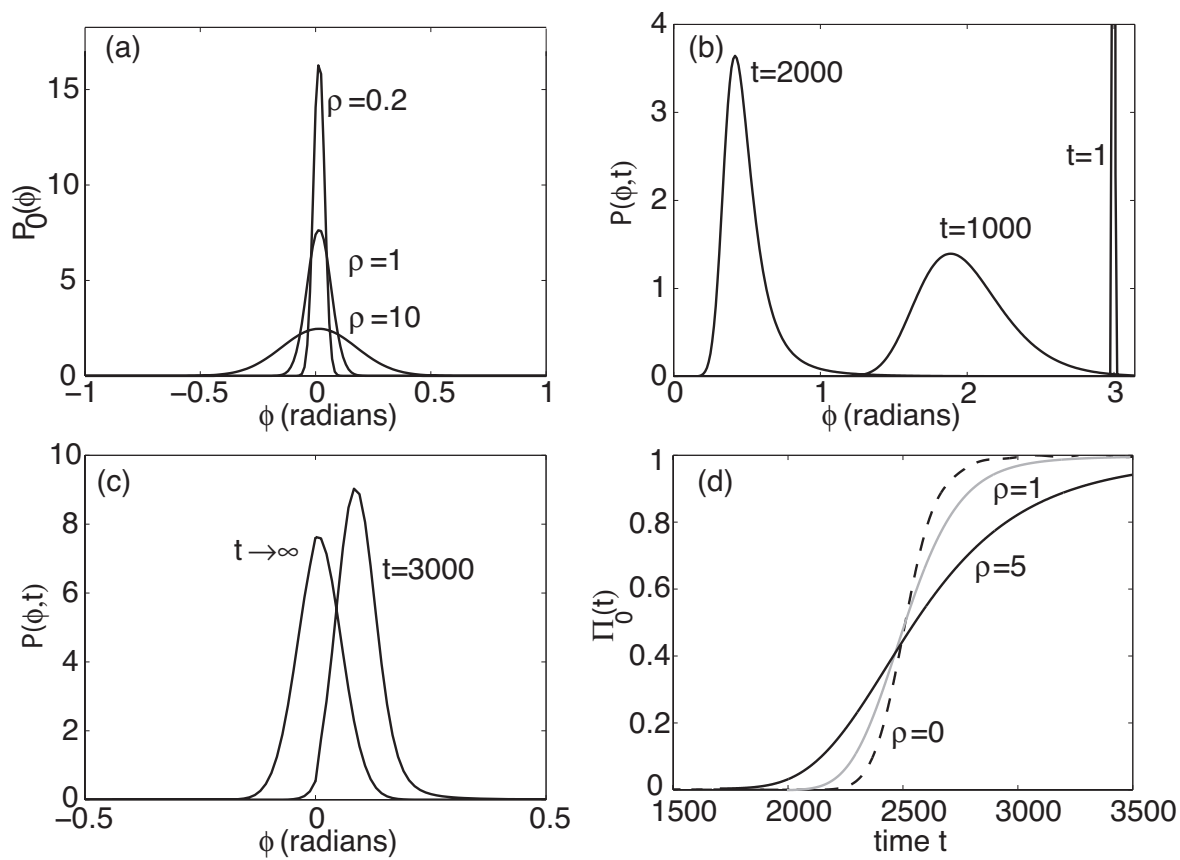

Fig. 7 Monostable case with correlated and uncorrelated noise. All parameters except $\rho$ are the same as Fig. 4. (a) Steady-state PDFs for various values of $\rho$. (b,c) For $\rho=1$, the $\mathrm{PDF}$ is plotted at several points in time, starting from the initial condition $\phi_{0}=3$ radians. (c) Plot of $\Pi_{0}(t)=\int_{-0.2}^{0.2} p(\phi, t) d \phi$ against time for various values of $\rho$. ( $\rho=0$ is the case without uncorrelated noise.) 

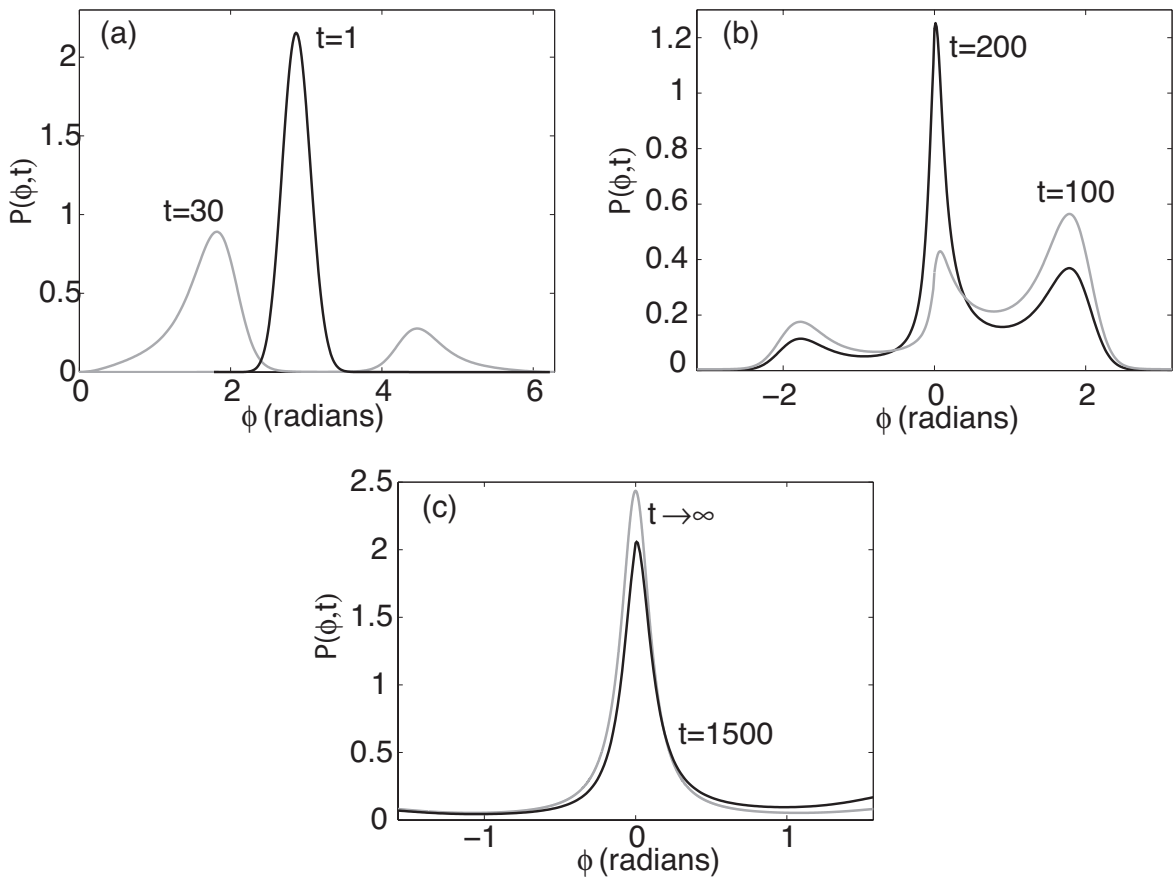

Fig. 8 Multistable case with correlated noise and a low level of uncorrelated noise $(\rho=$ 0.02). All other parameters are the same as Fig. 5. (a-c) Snapshots of the PDF $P(\phi, t)$ with $P(\phi, 0)=\delta\left(\phi-\phi_{0}\right)$ and $\phi_{0}=3$. (a) At relatively short times the PDF develops two peaks at the deterministic asynchronous states $\phi \approx 1.86$ and $2 \pi-1.86$. (b) At intermediate times the PDF develops a third peak at $\phi=0$. c) At longer times the PDF approaches the steady-state solution which is given by a single peak centered about the synchronous state.

Note that as the level of uncorrelated noise goes to zero $(\rho \rightarrow 0), K^{*}(0) \rightarrow 0$ and $P_{0}$ tends to a delta function at zero as in section 2 .

We now explore the effects of uncorrelated noise on synchronisation by numerically solving the FP equation (40). In the monostable case, see Fig. 7 , where both dispersal and correlated noise cause the system to synchronize irrespective of initial conditions, adding uncorrelated noise simply broadens the steady-state PDF to give a unimodal function centred at $\phi=0$ whose width increases with the level $\rho$ of uncorrelated noise. Moreover, when $\rho>$ 0 , partial synchrony occurs faster than the case of zero uncorrelated noise $(\rho=0)$, but it takes a longer time to reach higher levels of synchrony. In the multistable case, introducing a small amount of uncorrelated noise $(\rho=0.02)$ leads to a PDF with multiple peaks at intermediate times before converging to a unimodal steady-state centred about $\phi=0$, see Figs. 8(a-c). Increasing the level of uncorrelated noise $(\rho=1)$ causes the transient peaks to persist so that the steady-state PDF develops multiple peaks indicative of a stochastic bifurcation, see Fig. 9)a,b. Finally, adding a high amount of uncorrelated noise $(\rho=10)$ the steady-state PDF approaches a unform distribution, see Fig. 9c. 

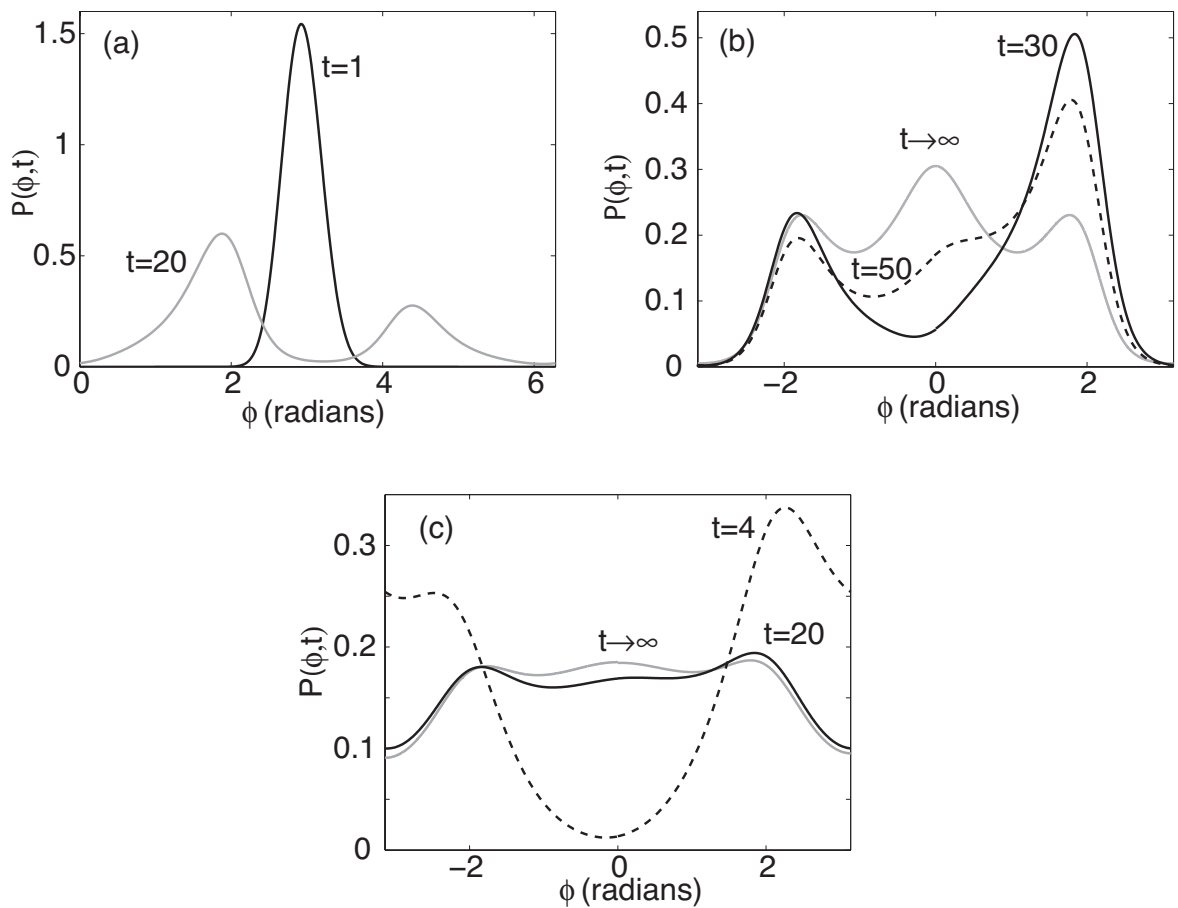

Fig. 9 (a,b) Same as Fig. 8 for an intermediate level of uncorrelated noise $(\rho=1)$. The PDF is plotted at various points in time to show the emergence of persistent peaks around the asynchronous states and convergence to a multistable steady state . c) At high levels of uncorrelated noise $(\rho=10)$, the steady-state distribution becomes more uniform.

It is also instructive to see how the steady-state probability of being in a neighborhood of the synchronous state $\phi=0$ or the asynchronous state $\phi=$ $\phi_{a}=1.86$ varies with $\rho$ and $\sigma$. Thus let $\Pi_{\phi}=\int_{\phi-\Delta}^{\phi+\Delta} P_{0}\left(\phi^{\prime}\right) d \phi^{\prime}$ with $\Delta=0.2$ be
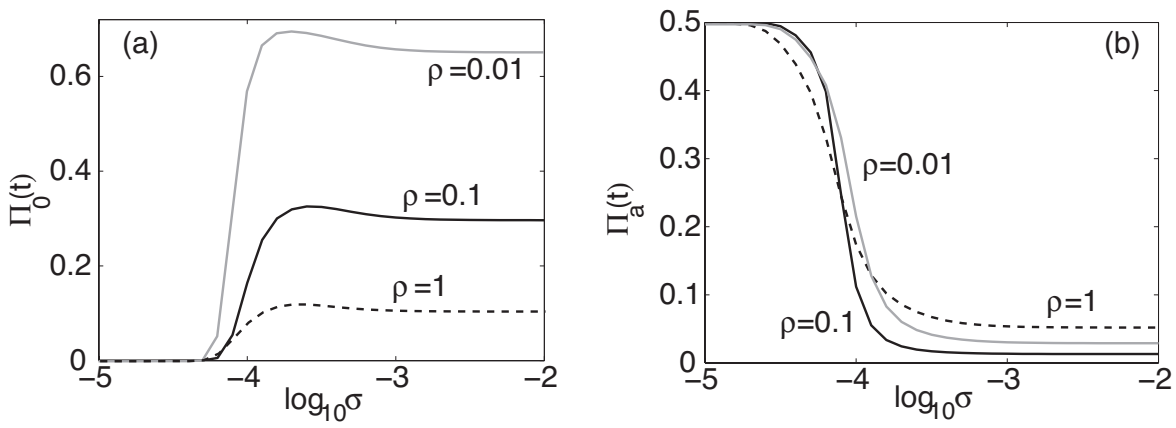

Fig. 10 Plots of $\Pi_{\phi}=\int_{\phi-0.2}^{\phi+0.2} P_{0}(\phi) d \phi$ against $\log \sigma$ for different values of $\rho$. (a) Synchronous state $\phi=0$; (b) Asynchronous state $\phi=\phi_{a} \approx 1.86$. Note that the maximum probability is 0.5 due to the symmetrical steady state at $\phi=2 \pi-1.85$. 
a measure of the degree of stochastic phase-locking around the phase $\phi$. In Fig. 10 we plot $\Pi_{0}$ and $\Pi_{\phi_{a}}$ as a function of the noise level $\sigma$ for various $\rho$. It can be seen that when the common noise source is dominant $\left(\sigma>10^{-4}, \rho \ll 1\right)$ there is a high probability that the oscillators are approximately synchronized with $\Pi_{0}>0.5$ and $\Pi_{a} \approx 0$. As the level of uncorrelated noise $\rho$ is increased for fixed $\sigma$, the degree of synchronization decreases but there is still a low probability of being in the asynchronous states. On the other hand, for sufficiently low levels of the common noise source $\left(\sigma<10^{-4}\right)$, the oscillators occupy one of the two asynchronous states with a probaility $\Pi_{a} \approx 0.5$, and this is insensitive to the level of uncorrelated noise. Interestingly, there is a sharp transition between synchrony and asynchrony as $\sigma$ increases for fixed $\rho$.

\section{Discussion}

In this paper we used the theory of noise-induced phase synchronization to analyze the effects of dispersal on the synchronization of a pair of predator-prey systems within a fluctuating environment. We first showed that in the case of common environmental noise, the oscillators ultimately synchronize. However, the approach to synchrony depends on whether or not there exist stable asynchronous states in the deterministic limit. We found that in the absence of asynchronous states, the time-dependent PDF was Gaussian-like with a width that first increased and then decreased as it moved towards the synchronous state. Moreover, the rate of stochastic synchronization varied approximately linearly with the initial phase difference between the two oscillators. On the other hand, if dispersal also supported asynchronous phase-locking, then the corresponding PDF exhibited a broad bimodal structure at intermediate times before ultimately converging to the synchronous state. This led to a sharp decrease in the rate of stochastic synchronisation as the initial phase difference increased from zero. We then investigated how these two distinct scenarios were affected by uncorrelated noise. In particular, we showed how uncorrelated noise can cause the transient peaks in the PDF associated with asynchronous states to become persistent, resulting in a multimodal steady-state PDF that is indicative of a stochastic bifurcation.

One obvious extension of our work would be to consider a metapopulation of $N$ predator-prey oscillators with both disperal and noise, as described by the system of equations (33) with the corresponding multivariate FP equation (38). This is a considerably more involved problem from an analytical perspective. Following previous studies of the Kuramoto model $[19,36]$, one could proceed by identifying appropriate macroscopic variables (for large $N$ ) that characterize the degree of synchrony of the system. A common choice is the complex amplitude defined according to

$$
R \mathrm{e}^{i \psi}=\frac{1}{N} \sum_{i=1}^{N} \mathrm{e}^{i \theta_{i}}
$$


The amplitude $R \in[0,1]$ with $R=1$ denoting complete synchrony and $R=0$ denoting complete asynchrony. In the case of common noise and dispersal, we expect the population to converge to a synchronous state just as for a pair of oscillators. One could then investigate numerically how the approach to synchrony $(R(t) \rightarrow 1$ as $t \rightarrow \infty)$ depends on the initial distribution of phases and the various phase-locked states in the deterministic limit. For large $N$ there could be a large number of such states associated with various forms of clustering. Including the effects of intrinsic noise would counter the synchronizing effects of dispersal and common noise, but characterizing the associated steady-state PDF for $N$ phases would be non-trivial.

Our results could provide an analytical background into various ecological experiments to determine the dominant factors behind the behaviour of ecosystems - for example, controlled experiments could be done in phytoplanktonzooplankton systems, with different levels of environmental fluctuations and ease of dispersal between populations, that could be compared with our results to find out which parameter regime the system is in. In the field, population data could be processed in a similar manner - for instance, constant time-lags between the peaks of populations of adjacent patches would be indicative of a constant phase difference, showing that the asynchronous dispersal-induced state was present; alternatively, switching between this constant delay and synchronization would demonstrate multistability.

\section{Acknowledgements}

This publication was based on work supported in part by the National Science Foundation (DMS-1120327) and by Award No KUK-C1-013-04, made by the King Abdullah University of Science and Technology (KAUST).

\section{References}

1. P. C. Bressloff and Y.M. Lai. Stochastic synchronization of neuronal populations with intrinsic and extrinsic noise. J. Math. Neurosci. 1:2 (2011).

2. A. Colombo, F. Dercole and S. Rinaldi. Remarks on metacommunity synchronization with application to prey-predator systems. The American Naturalist. 171:430-442 (2008).

3. D. J. D. Earn, P. Rohani and B. T. Grenfell. Persistence, chaos and synchrony in ecology and epidemiology. Proc R Soc Lond Ser B: Biol Sci 265: 7-10 (1998).

4. G. B. Ermentrout and N. Kopell. Multiple pulse interactions and averaging in coupled neural oscillators. J. Math. Biol. 29:195-217 (1991).

5. R. F. Galan, G. B. Ermentrout and N. N. Urban. Correlation-induced synchronization of oscillations in olfactory bulb neurons. J. Neurosci. 26:3646-3655 (2006).

6. R. F. Galan, G. B. Ermentrout and N. N. Urban. Optimal time scale for spike-time reliability: theory, simulations and expeiments. J. Neurophysiol. 99:277-283 (2008).

7. A. Ghandi, S. Levin and S. Orszag 'Critical slowing down' in time-to-extinction: an example of critical phenomena in ecology. J. Theor. Biol. 192, 363-76 (1998).

8. A. Ghandi, S. Levin and S. Orszag. Moment expansions in spatial ecological models and moment closure through Gaussian approximation. Bull. Math. Biol. 62, 595-632 (2000).

9. C.W. Gardiner, Stochastic methods: a handbook for the natural and social sciences. 4th ed. (Springer, NY, 2009). 
10. D. S. Goldobin and A. Pikovsky. Synchronization and desynchronization of selfsustained oscillators by common noise. Phys. Rev. E, 71: 045201 (2005).

11. E.E. Goldwyn and A. Hastings. When can dispersal synchronize populations? Theoretical Population Biology 73:395-402 (2008).

12. E.E. Goldwyn and A. Hastings. Small heterogeneity has large effects on synchronisation of ecological oscillators. Bull. Math. Biol. 71:130-144 (2009).

13. E.E. Goldwyn and A. Hastings. The roles of the Moran effect and dispersal in synchronizing oscillating populations. J. Theor. Biol. 289:237-246 (2011).

14. I. Hanski. Metapopulation dynamics. Nature 396:41-49 (1998).

15. P. J. Hudson and I. M. Cattadori. The Moran effect: a cause of population synchrony. Trends Ecol. Evol. 14: 1-2 (1999).

16. V. A. A. Jansen and A. L. Lloyd. Local stability analysis of spatially homogeneous solutions of multi-patch systems. J. Math. Biol. 41: 232-252 (2000).

17. C.A. Klausmeier. Successional state dynamics: A novel approach to modeling nonequilibrium foodweb dynamics, J. Theor. Biol. f262:585-595 (2010).

18. A. Hastings. Population Biology: Concepts and Models. Springer-Verlag, New York (1997).

19. Y. Kuramoto, Chemical oscillations, waves and turbulence. (Springer, Berlin, 1984).

20. Y.M. Lai, J. Newby and P. C. Bressloff. Effects of demographic noise on the synchronization of metacommunities by a fluctuating environment. Phys. Rev. Lett, 107:118102 (2011).

21. A. Liebhold and W. D. Koenig and O. N. Bjornstad. Spatial synchrony in population dynamics. Ann. Rev. Ecol. Evol. Syst. 35: 467-490 (2004)

22. C. Ly and G. B. Ermentrout. Synchronization of two coupled neural oscillators receiving shared and unshared noisy stimuli. J. Comput. Neurosci., 26:425-443 (2009).

23. A.J. McKane and T.J. Newman. Stochastic models in population biology and their deterministic analogs. Phys. Rev. E 70: 041902 (2004).

24. A.J. McKane and T.J. Newman. Predator-prey cycles from resonant amplification of demographic stochasticity. Phys. Rev. Lett. 94: 218102 (2005)

25. Mainen ZF, Sejnowski TJ. Reliability of spike timing in neocortical neurons. Science 268:1503-1506 (1995).

26. S. Marella and G. B. Ermentrout. Class-II neurons display a higher degree of stochastic synchronization than class-I neurons. Phys. Rev. E 77:41918 (2008)

27. E. McCauley, W. G. Wilson. and A. M. Roos. Dynamics of age-structured predator-prey interactions: Individual based models and population level formulations. Am. Nat. 142, 412-442 (1993).

28. P. A. P. Moran. The statistical analysis of the Canadian lynx cycle. II. Synchronisation and meteorology. Australian Journal of Zoology 1:291-298 (1953).

29. H. Nakao, K. Arai, and Y. Kawamura. Noise-induced synchronization and clustering in ensembles of uncoupled limit cycle oscillators. Phys. Rev. Lett., 98: 184101 (2007).

30. L. M. Pecora and T. L. Carroll. Master stability functions for synchronized coupled systems. Phys. Rev. Lett. 80: 2109-2112 (1998).

31. A. S. Pikovsky. Synchronization and stochastization of an ensemble of autogenerators by external noise Radiophys. 27:576-581 (1984).

32. E Renshaw, Modelling Biological Populations in Space and Time. Cambridge University Press, 1993.

33. P. Rohani, D. J. D. Earn and B. T. Grenfell. Opposite patterns of synchrony in sympatric disease metapopulations. Science 286:968-971 (1999).

34. A. M. Roos, E. McCauley and W. G. Wilson. Mobility versus Density-Limited PredatorPrey Dynamics on Different Spatial Scales. Proc. R. Soc. London B, 246:117-122 (1991).

35. M. L. Rosenzweig and R. H. MacArthur. Graphical representation and stability conditions of predator-preyinteractions. Am. Nat. 97:209-223 (1963).

36. S. H. Strogatz. From Kuramoto to Crawford: exploring the onset of synchronization in populations of coupled oscillators. Physica D 143: 1-20 (2000).

37. J. N. Teramae and D. Tanaka. Robustness of the noise-induced phase synchronization in a general class of limit cycle oscillators. Phys. Rev. Lett. 93:204103 (2004).

38. J. N. Teramae, H. Nakao and G. B. Ermentrout. Stochastic phase reduction for a general class of noisy limit cycle oscillators. Phys. Rev. Lett., 102:194102 (2009). 
39. N G Van Kampen. Stochastic processes in physics and chemistry. North-Holland, Amsterdam, 1992.

40. D. A. Vasseur and J. W. Fox. Phase-locking and environmental fluctuations generate synchrony in a predator-prey community. Nature, 460: 1007-1010 (2009).

41. K. Yoshimura and K. Arai. Phys. Rev. Lett. 101154101 (2008). 



\section{RECENT REPORTS}

12/14 Wildlife disease elimination and density dependence

Potapova

Merrill

Lewis

12/15 Spreading Speed, Traveling Waves, and Minimal Domain Size in Impulsive Reaction-diffusion Models

Lewis

$\mathrm{Li}$

12/16 MCMC methods for functions modifying old algorithms to make them faster

Cotter

Roberts

Stuart

White

12/17 Weyl Geometry and the Nonlinear Mechanics of Distributed Point Yavari Defects

Goriely

12/18 A note on oblique water entry

Moore

Howison

Ockendon

Oliver

12/19 Calculus on surfaces with general closest point functions

März

Macdonald

12/20 Multiple equilibria in a simple elastocapillary system

Taroni

Vella

12/21 Multiphase modelling of vascular tumour growth in two spatial dimensions

Hubbard

Byrne

Hale

Trefethen

12/23 Moment-based formulation of NavierMaxwell slip boundary con-

Reis ditions for lattice Boltzmann simulations of rarefied flows in microchannels

12/24 Correspondence between one- and two-equation models for solute transport in two-region heterogeneous porous media

Davit

Wood

Debenest

Quintard

12/25 Rolie-Poly fluid flowing through constrictions: Two distinct insta- Reis bilities

Wilson

12/26 Age related changes in speed and mechanism of adult skeletal muscle stem cell migration

Collins-Hooper

Woolley

Dyson

Patel

Potter

Baker

Gaffney

Maini

Dash

Patel

12/27 The interplay between tissue growth and scaffold degradation in

ODea engineered tissue constructs 
12/28 Non-linear effects on Turing patterns: time oscillations and Aragon chaos.

Barrio

Woolley

Baker

Maini

12/29 Colorectal Cancer Through Simulation and Experiment

Kershaw

Byrne

Gavaghan

Osborne

12/30 A theoretical investigation of the effect of proliferation and adhe-

Mirams sion on monoclonal conversion in the colonic crypt

Fletcher

Maini

Byrne

12/31 Convergent evolution of spiny mollusk shells points to elastic energy minimum

Chirat

Moulton

Shipman

Goriely

12/32 Three-dimensional oblique water-entry problems at small dead-

Moore rise angles

Howison

Ockendon

Oliver

12/33 Second weak order explicit stabilized methods for stiff stochastic

Abdulle differential equations

Vilmart

Zygalakis

12/34 The sensitivity of Graphene 'Snap-through' to substrate geometry Wagner

Vella

12/35 The physics of frost heave and ice-lens growth

Peppin

Style

12/36 Finite Element Simulation of Dynamic Wetting Flows as an Inter-

Sprittles face Formation Process

Shikhmurzaev

12/37 The Dynamics of Liquid Drops and their Interaction with Solids of

Sprittles Varying Wettabilities

Copies of these, and any other OCCAM reports can be obtained from:

Oxford Centre for Collaborative Applied Mathematics

Mathematical Institute

24 - 29 St Giles'

Oxford

OX1 3LB

England 
www.maths.ox.ac.uk/occam 